\title{
Nota Técnica: \\ Ductilidad del acero inoxidable bajo en níquel para estructuras de hormigón armado
}

\author{
Technical Note: \\ Ductility in a new low nickel stainless steel for reinforced concrete
}

\author{
A. Cobo(*), D. M. Bastidas ${ }^{(* *)}$, M. N. González(*), E. Medina(*), J. M. Bastidas(**)
}

Recepción/Received: 15-I-10

Aceptación/Accepted: 1-XII-10

\section{RESUMEN}

En este trabajo se presentan los diagramas tensióndeformación de un nuevo acero inoxidable con bajo contenido en níquel, un inoxidable convencional AISI 304 y un acero al carbono de uso común en estructuras de hormigón armado. Dicha ductilidad se ha estudiado determinando la tensión máxima $\left(f_{\max }\right)$, la tensión en el límite elástico $\left(f_{\mathrm{y}}\right)$ y la deformación bajo carga máxima $\left(\varepsilon_{\max }\right)$. Los tres materiales se han evaluado utilizando criterios aceptados internacionalmente, como son el índice $p$ (capacidad de rotación plástica), el índice $A^{*}$ (área plástica de endurecimiento) y el índice de tenacidad $I_{d}$ (energía total absorbida en el punto de alargamiento bajo carga máxima), los resultados obtenidos se han comparado con los aceros convencionales de armaduras 500SD, $500 \mathrm{~N}$ y $500 \mathrm{H}(\mathrm{EC}-2)$.

Palabras clave: acero bajo en níquel, ductilidad, armaduras, acero equivalente, estructuras de hormigón armado.

\section{SUMMARY}

This paper discusses the stress-strain curves for a new low nickel stainless steel, a conventional AISI 304 stainless steel and a carbon steel commonly used in reinforced concrete structures. Ductility was studied in terms of ultimate tensile strength $\left(f_{\text {max }}\right)$, elastic limit $\left(f_{y}\right)$ and total elongation at maximum force [ultimate strain; uniform elongation] $\left(\varepsilon_{\max }\right)$. The three materials were assessed with internationally accepted criteria, such as plastic rotational capacity, necking region and the toughness index (total energy absorbed at uniform elongation). The findings were compared to the properties of three types of conventional reinforcing steel: 500SD, 500N and 500H (EC-2).

Keywords: low nickel steel, ductility, reinforcement, equivalent steel, reinforced concrete structures.

\footnotetext{
(*) Universidad Politécnica de Madrid (Madrid, España).

(**) CENIM-CSIC (Madrid, España).
} 


\section{INTRODUCCIÓN}

El hormigón armado combina la elevada resistencia a la compresión del hormigón y la excelente resistencia a la tracción del acero (1). Sin embargo, el fallo prematuro de las barras de acero corrugado tiene un grave efecto sobre la durabilidad de las estructuras de hormigón armado (EHA). Debido principalmente a la presencia de cloruros, a la carbonatación del hormigón o a la existencia de malas prácticas durante el proceso de construcción, facilitando como consecuencia el ingreso de agua y reactivos químicos perjudiciales para la estructura, y provocando que el acero embebido en hormigón pueda experimentar pérdida de sus estado pasivo a velocidades que provoquen un elevado daño en unos pocos años. Éste es el caso de los pilares de puentes de hormigón en contacto con sales de deshielo, plataformas sumergidas y estructuras expuestas a ambiente marinos en climas cálidos, o en casos en los que exista contaminación por cloruros dentro del propio hormigón (2). La predicción de vida útil en servicio para puentes de hormigón es de 100 años según la Instrucción de Hormigón Estructural (EHE08), esto significa que en la actualidad la durabilidad de las EHA es un factor determinante.

Existen varias alternativas que han sido propuestas con objeto de proteger las armaduras de acero al carbono en ambientes agresivos: a) El uso de refuerzos con recubrimientos orgánicos tipo epoxy, el cual presenta varios problemas que no han sido resueltos en hasta el momento como son la adhesión entre el recubrimiento orgánico y el substrato metálico y por otro lado el daño y deterioro del recubrimiento orgánico durante el transporte, manipulación y procesos de ensamblaje (3). b) La aplicación de recubrimientos resistentes al agua y sellantes, o el uso de armaduras poliméricas de fibra de carbono (4). c) El uso de barras de acero galvanizado y la adición durante el mezclado de inhibidores de corrosión. Los recubrimientos galvanizados ofrecen una barrera física que evita el contacto de los agentes agresivos con el substrato de acero, actuando el zinc como ánodo de sacrificio y protegiendo así el acero frente a la corrosión (5). El cromado ha sido utilizado de forma generalizada en la industria como pretratamiento para mejorar la resistencia frente a la corrosión del acero galvanizado, sin embargo, en la actualidad no se permite la utilización de cromo hexavalente debido a su toxicidad y sus efectos cancerígenos. Las Normativas de la Unión Europea 2000/53/EC y 2005/438/EC limitan el uso de compuestos de cromo (VI) en la industria automovilística, también se ha restringido el contenido de cromo (VI) en la composición del cemento Pórtland. Los iones metálicos de tierras raras, como el cerio o el lantano, han sido propuestos como alternativas a los recubrimientos de cromo. El nitrato de cerio ha sido estudiado con éxito como protector frente a la corrosión de substratos de acero galvanizado tanto como inhibidor de corrosión (6), como capa de conversión (7), así como elemento dopante para capas finas y recubrimientos de tipo sol-gel (8). Las propiedades del cerio como inhibidor de la corrosión tienen como fundamento un mecanismo de inhibición de tipo catódico atribuido a la precipitación de capas de óxidos e hidróxidos de cerio en sitios catódicos (9). La protección frente a la corrosión de substratos de acero galvanizado en presencia de iones lantano se atribuye a la formación de una capa fina de $\mathrm{La}_{2} \mathrm{O}_{3}$, $\mathrm{La}(\mathrm{OH})_{3}$, conjuntamente con pequeñas cantidades de $\mathrm{Zn}(\mathrm{OH})_{2}$ y $\mathrm{ZnO}$. d) La aplicación de métodos de rehabilitación electroquímica: dentro de los cuales, a) la protección catódica ha sido la única alternativa provada que paraliza de manera efectiva la corrosión en pilares de puentes contaminados con cloruros, no dependiendo ésta del contenido total de cloruros (10); b) la realcalinización electroquímica puede repasivar el acero cuando el estado de corrosión es todavía incipiente, pero no puede repasivar las armaduras de acero cuando éstas han alcanzado un estado avanzado de corrosión (11-13). Por otro lado, c) la extracción electroquímica de cloruros es otro de los procedimientos de rehabilitación electroquímica, aunque sigue provocando controversia al evaluar su eficiencia (3). Actualmente, los estudios relacionados con la extracción electroquímica de cloruros se centran en la especificación de sus efectos sobre los productos de corrosión del acero, la permeabildad del mortero y los cambios microestructurales que tienen lugar en la matriz de mortero (14). Finalmente, d) el uso de armaduras de acero inoxidable despierta gran interés dada su elevada resistencia frente a la corrosión en una gran variedad de ambientes. Las propiedades de autoregeneración de su capa pasiva y su mínimo trabajo de mantenimiento lo convierte en una alternativa muy atractiva para la fabricación de EHA (15).

Los aceros inoxidables austeníticos y dúplex (austeníticoferrítico) son los que ofrecen una buena combinación entre propiedades de resistencia frente a la corrosión y mecánica (tensión y tenacidad), así como viabilidad económica y disponibilidad para ser considerado su uso en EHA. Tradicionalmente, los aceros inoxidables ferríticos son los más económicos.

Existen diversas EHA fabricadas con acero inoxidable, entre ellas destacan: el embarcadero del Puerto de Progreso en Yucatán (Golfo de México), construido entre el 1937 y el 1941, donde su última inspección en 1998 reveló la práctica ausencia de corrosión (16); el puente de la autopista de Cala Galdana en la isla de Menorca (España); el puente del Padre Arrupe que une el museo Guggenheim con la Universidad de Deusto en Bilbao (España); el puente Millennium en York (Reino Unido); el puente de Stonecutters en Hong Kong; y el puente del Tercer Milenio sobre el río Ebro en Zaragoza (España), siendo éste el puente suspendido sobre arcos de hormigón más largo del mundo con una longitud de 270 m.

La experiencia en el uso de armaduras de acero inoxidable en EHA es mayoritariamente con acero inoxidable austenítico. El 
níquel está sujeto a notables fluctuaciones de precio debido a factores de oferta y demanda del mercado, habiendo alcanzado en los últimos años precios sin precedentes, afectando de forma directa el coste del acero inoxidable austenítico, ya que su contenido en níquel es del $8 \%$ (17). El precio de las barras de acero inoxidable corrugado es entre 5 y 8 veces superior al de las de acero al carbono (18), siendo ésta la principal razón que limita el uso del acero inoxidable en EHA. Sin embargo, el uso de armaduras de acero inoxidable representa tan sólo un incremento del $10 \%$ del coste total del proyecto de construcción, y se ha estimado que su amortización puede reducir el coste inicial en un $50 \%$ si consideramos el incremento de tiempo de vida útil en servicio, el cual puede ser superior a 120 años para puentes en zonas costeras (19).

Las normativas españolas de UNE clasifican los tipos de acero, dimensiones, características geométricas y los métodos de ensayo para barra de acero inoxidable corrugado (20). Las normativas de la ASTM y "British Standards" para EHA describen la utilización de acero inoxidable en armaduras de hormigón $(21,22)$. Sin embargo, estas normativas están basadas en especificaciones para el acero al carbono, por lo que existe poco conocimiento sobre el comportamiento de EHA fabricadas con acero inoxidable.

El objetivo de este trabajo es analizar las propiedades de ductilidad de un nuevo tipo de acero inoxidable con bajo contenido en níquel, el cual ofrece ventajas desde el punto de vista económico y medioambiental dada la reducción del contenido en níquel. Se analizarán los criterios propuestos por Cosenza y col., Creazza y col. y Ortega (23-25) y se evaluará la utilización en EHA del nuevo acero inoxidable bajo en níquel, se estudiarán a efectos comparativos el acero inoxidable AISI 304 y un acero al carbono convencional B 500S.

\section{CARACTERÍSTICAS DE LAS ARMADURAS Y DUCTILIDAD}

En la actualidad, la aplicación de métodos de cálculo de EHA no lineales o con redistribución limitada, de acuerdo a la Instrucción de Hormigón Estructural EHE-08 (26), exige que las secciones de las estructuras posean una determinada capacidad de giro. Para ello es necesario que la armadura cumpla una serie de condiciones que globalmente se definen bajo el concepto de ductilidad (27). Es por ello que todas las normas europeas de hormigón estructural enfatizan la necesidad del empleo de aceros con unas características mínimas de ductilidad.

La Figura 1 presenta el diagrama tensión ( $f$ ) deformación $(\varepsilon)$, del nuevo acero inoxidable bajo en níquel propuesto en este trabajo, el cual no presenta escalón de cedencia. La forma habitual de cuantificar la ductilidad del acero es mediante los siguientes parámetros obtenidos a partir del diagrama tensión deformación de un ensayo de tracción. La relación entre la carga de rotura y el límite elástico $\left(f_{\text {max }} / f_{\mathrm{y}}\right)$. Indica la reserva de resistencia que le queda al acero una vez alcanzado el límite elástico. El acero se dimensiona para alcanzar el límite elástico, cuanto mayor sea la relación, mayor seguridad presenta a rotura. Por otro lado, se determina la deformación bajo carga máxima $\left(\varepsilon_{\max }\right)$. Cuanto mayor es este valor, más se puede deformar el acero y más ductilidad presenta. Desde un punto de vista de cálculo de estructuras, la relación $\left(f_{\text {max }} / f_{y}\right)$ está generalmente acotada por un límite superior e inferior. Se define por tanto un factor de seguridad $\left(F_{\mathrm{S}}\right)$, como el inverso de la relación anterior, siendo $F_{\mathrm{s}}=f_{\text {max }} / f_{\mathrm{y}}$, el cual está comprendido entre $0.70 \leq F_{s} \leq 0.80$.

La Tabla 1 muestra las características de ductilidad mínimas exigidas para los cuatro tipos de acero al carbono

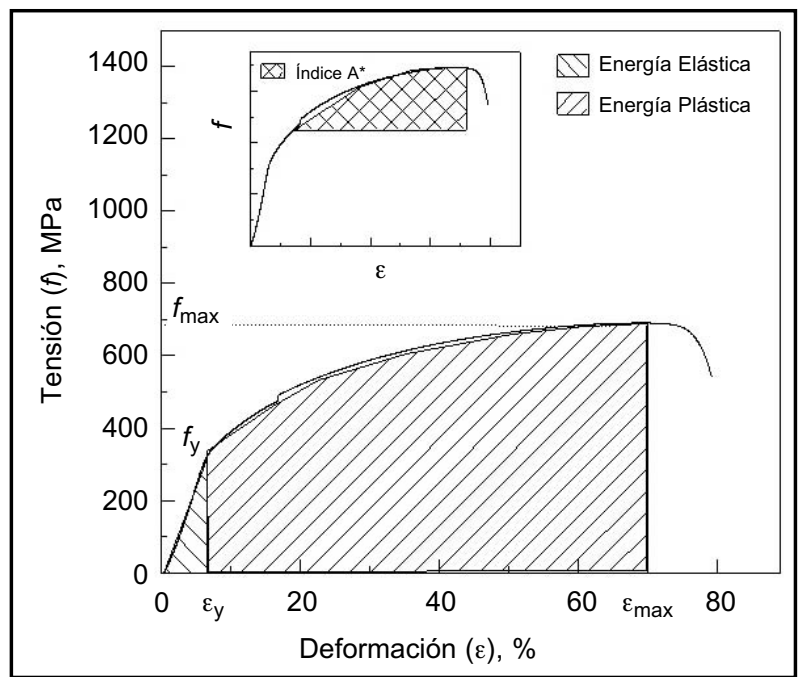

Figura. 1. Diagrama tensión deformación de una barra del nuevo acero inoxidable bajo en níquel, sin escalón de cedencia.

Tabla 1

Parámetros de ductilidad mínimos exigidos para barras de acero de acuerdo a la Instrucción de Hormigón Estructural, EHE-08 (26).

\begin{tabular}{|c|c|c|c|c|}
\hline \multirow{2}{*}{ Parámetro } & \multicolumn{3}{|c|}{ Ductilidad } \\
\cline { 2 - 5 } & \multicolumn{2}{|c|}{ Aceros tipo B 400S, B 500S } & 480 & \multicolumn{2}{c|}{ Aceros tipo B 400SD, B 500SD } \\
\hline$f_{\max } \mathrm{N} \mathrm{mm}^{-2}$ & 440 & 550 & 400 & 575 \\
\hline$f_{\mathrm{y},} \mathrm{N} \mathrm{mm}^{-2}$ & 400 & 500 & 7.5 & 7.5 \\
\hline$\varepsilon_{\max }$ bar, $\%$ & 5.0 & 5.0 & 10.0 & 10.0 \\
\hline$\varepsilon_{\max }$ roll, \% & 7.5 & 7.5 & $1.20-1.35$ & $1.20-1.35$ \\
\hline$f_{\max } / f_{\mathrm{y}}$ & 1.05 & 1.05 & \\
\hline
\end{tabular}


contemplados en la Instrucción de Hormigón Estructural EHE-08 (26). Los aceros según presenten o no características especiales de ductilidad (SD y S) y en función del valor de tensión en el límite elástico $\left(f_{\mathrm{y}}\right) 400$ y $500 \mathrm{~N}$ $\mathrm{mm}^{-2}$, se clasifican de acuerdo a dos parámetros: la deformación bajo carga máxima $\left(\varepsilon_{\max }\right)$ y la relación entre la tensión máxima y la tensión en el límite elástico $\left(f_{\text {max }} / f_{\mathrm{y}}\right)$.

La Tabla 2 muestra las características mínimas de ductilidad para armaduras de acero con valores de ductilidad normales $(\mathrm{N})$ o altos $(\mathrm{H})$ según los códigos europeos MC90 (28) y EC-2 (29).

\section{CONCEPTO DE ACERO EQUIVALENTE}

La Instrucción de Hormigón Estructural EHE-08 así como los códigos europeos MC-90 y EC-2 comprenden una clasificación de las armaduras de acero en función de la ductilidad, la cual se define en función de dos parámetros $(26,28,29)$ : la deformación bajo carga máxima $\left(\varepsilon_{\max }\right)$ y la relación $\left(f_{\max } / f_{\mathrm{y}}\right)$ entre la tensión máxima $\left(f_{\max }\right)$ y la tensión en el límite elástico $\left(f_{\mathrm{y}}\right)$.

En el caso de que un determinado acero no cumpliese con una de las especificaciones, aunque quedase cerca de alcanzarla, y pese a que para la otra condición el cumplimiento se realizase holgadamente, la aplicación estricta de la norma supondría la eliminación de ese acero, a pesar de que posiblemente su ductilidad fuese superior a la que tendría un acero que cumple estrictamente los dos indicadores.

Desde el Grupo de Trabajo de Ductilidad del Comité Europeo Internacional del Hormigón se ha desarrollado el concepto de acero equivalente que se define como un acero que da las mismas prestaciones de ductilidad que otro que cumple con las especificaciones mínimas de los códigos $\left(\varepsilon_{\max }\right.$ y $f_{\max } / f_{\mathrm{y}}$ ) pero no alcanzando uno de los valores anteriores y sobrepasando el otro (30).

Actualmente existen en la literatura tres criterios de acero equivalente obtenidos por Cosenza y col., Creazza y col. y Ortega.

El concepto de acero equivalente de Cosenza y col. se basa en la capacidad de rotación de una sección de una viga de una EHA (23), se define por tanto que dos aceros son equivalentes si y solo si producen la misma capacidad de giro en una sección de hormigón armado. Este acero estará definido únicamente por dos parámetros $\left(\varepsilon_{\max } \mathrm{y}\right.$ $f_{\text {max }} / f_{\mathrm{y}}$ ). Después de realizar un extenso análisis matemático para evaluar la capacidad de rotación de una sección, Cosenza y col. obtuvieron que ésta era proporcional a un parámetro " $p$ ". Este parámetro " $p$ " puede ser utilizado para definir el criterio de equivalencia entre diferentes aceros de refuerzo (31) [1]:

$$
p=\varepsilon_{\max }^{0.73}\left(\frac{f_{\max }}{f_{y}}-1\right)^{0.92}=\varepsilon_{\max }^{0.75}\left(\frac{f_{\max }}{f_{y}}-1\right)^{0.90}
$$

Creazza y col., propusieron el valor del área $\left(A^{*}\right)$ encerrada por la curva tensión deformación desde $f_{y}$ a $f_{\text {max }}$ la cual incorpora cuatro valores (24), la horizontal que pasa por $f_{y}$ y la vertical que pasa por $f_{\text {max }}$ la deformación bajo carga máxima $\varepsilon_{\max } y$ la deformación en el límite elástico $\varepsilon_{y}$, ver Figura 1 . El índice $A^{*}$ se define como el trabajo de deformación del material durante la fase de endurecimiento e incorpora el concepto de ductilidad, ofreciendo un único parámetro que comprende a $\varepsilon_{\max }$ y $f_{\max } / f_{\mathrm{y}}, \mathrm{y}$ que puede usarse como indicador de la ductilidad [2]:

$$
A^{*}=\frac{2}{3}\left(f_{\max }-f_{y}\right)\left(\varepsilon_{\max }-\varepsilon_{y}\right)
$$

El parámetro anterior depende del valor del límite elástico, aceros con los mismos valores de $\mathrm{f}_{\mathrm{s}} / \mathrm{f}_{\mathrm{y}}$ y $\varepsilon_{\text {max }}$, pero aceros con el límite elástico más alto, poseen un área $A^{*}$ superior, a pesar de que los indicadores que miden habitualmente la ductilidad son los mismos. Para eliminar la influencia del límite elástico en el parámetro que indica el acero equivalente, Ortega propone como indicador el Índice de Tenacidad $\left(\mathrm{I}_{d}\right)(25)$, que se obtiene como el cociente entre la tenacidad $(T)$ y la energía elástica (EE) obtenidas en el ensayo de tracción, siendo la tenacidad definida como $\mathrm{T}=\mathrm{EE}+\mathrm{PE}$, donde $\mathrm{PE}$ es la energía plástica [3]:

$$
I_{d}=\frac{T}{E E}=1+\left(1+\frac{f_{\max }}{f_{y}}\right)\left(\frac{\varepsilon_{\max }}{\varepsilon_{y}}-1\right)
$$

El índice de tenacidad $\mathrm{I}_{d}$ presenta la ventaja de ser un parámetro adimensional. Este criterio mide la energía absorbida por unidad de volumen durante el proceso de deformación de acero. La energía total absorbida hasta la rotura es el área comprendida en el diagrama tensión deformación, ver Figura 1, siendo la suma de la energía elástica (EE) y la energía plástica (PE).

Tabla 2

Parámetros de ductilidad mínimos exigidos para barras de acero de acuerdo a los códigos europeos CM-90 (28), y EC-2 (29).

\begin{tabular}{|c|c|c|}
\hline \multirow{2}{*}{ Parámetro } & \multicolumn{2}{|c|}{ Ductilidad } \\
\cline { 2 - 3 } & Aceros con ductilidad Normal (N) & Aceros con ductilidad Alta (H) \\
\hline$\varepsilon_{\max } \%$ & 2.5 & 5.0 \\
\hline$f_{\max } / f_{\mathrm{y}}$ & 1.05 & 1.08 \\
\hline
\end{tabular}




\section{MATERIALES Y MÉTODOS}

Se han ensayado tres tipos de acero: el nuevo acero inoxidable con bajo contenido en níquel, un acero inoxidable austenítico AISI 304 y un acero al carbono convencional B 500S. La Tabla 3 muestra la composición química de los tres aceros ensayados. El ensayo de tracción fue realizado con un equipo MicroTest (100 kN).

\section{RESULTADOS Y DISCUSIÓN}

En las Figuras 1 y 2 se muestran los diagramas tensión ( $\sigma$ ) deformación $(\varepsilon)$ para los tres aceros estudiados, el nuevo acero inoxidable bajo en níquel, el AISI 304 y el acero al carbono B 500S. La Tabla 4 muestra las propiedades mecánicas de los tres aceros e indica el valor mínimo exigido para el acero B500SD según la Instrucción de Hormigón Estructural EHE-08 (26), para acero con ductilidad normal (500N) o alta $(500 \mathrm{H})$ y tensión del límite elástico $\left(\sigma_{y}\right)$ de $500 \mathrm{~N}$ $\mathrm{mm}^{-2}$ en los códigos europeos MC-90 y EC-2 $(28,29)$, así como los parámetros de acero equivalente de acuerdo a Cosenza y col. (23), Creazza y col. (24) y Ortega (25).

Han sido utilizados dos métodos para evaluar los diferentes aceros, acero inoxidable bajo en níquel, AISI 304 y acero al carbono B 500S, desde un punto de vista de la ductilidad. El primero contempla la relación $f_{\text {max }} / f_{y}$ y el valor de $\varepsilon_{\max }$ para los tres aceros, y compara estos valores con los valores mínimos exigidos en la Instrucción EHE-08 (26) y del mismo modo con los valores comprendidos en los códigos europeos MC-90 y EC-2 $(28,29)$. El segundo método aplica el criterio de acero equivalente ( $p, y$ los índices $A^{*} y$ $I_{d}$ ), comparando estos valores con los que se obtendrían aplicando los criterios de Cosenza y col., Creazza y col. y Ortega para los aceros usualmente utilizados 500SD, 500N o $500 \mathrm{H}$ (23-25). El criterio de acero equivalente ofrece un único parámetro para evaluar la ductilidad, siendo esta información de gran relevancia para comparar diferencias de ductilidad entre diferentes tipos de aceros (32).

En la Figura 3 se muestra la relación $\left(f_{\text {max }} / f_{\mathrm{y}}\right)$ resultante para los tres tipos de acero ensayados, y el valor inferior límite indicado en la Instrucción EHE-08 para el acero 500SD (26), conjuntamente con los valores indicados por los códigos europeos CM-90 y EC-2 para los aceros $500 \mathrm{~N}$ y $500 \mathrm{H}(28,29)$. La Figura 4 presenta el valor de la deformación bajo carga máxima $\left(\varepsilon_{\max }\right)$ para los tres aceros ensayados y el valor inferior límite indicado en la Instrucción EHE-08 para el acero 500SD, además de los valores indicados por los códigos europeos CM-90 y EC-2 para los aceros $500 \mathrm{~N}$ y $500 \mathrm{H}$. Como puede deducirse de las Figuras 3 y 4, los tres aceros satisfacen completamente las exigencias de la Instrucción EHE-08 así como de los códigos europeos CM-90 y EC-2, cabe destacar que incluso superan notablemente los valores exigidos de ductilidad, aún en la situación más desfavorable, es decir, el acero tipo 500SD con valores mínimos de $f_{\text {max }} / f_{\mathrm{y}}$ y $\varepsilon_{\max }$ de 1.20 y $10 \%$, respectivamente. Los valores obtenidos para el acero al carbono $\left(f_{\max } / f_{\mathrm{y}}=1.26 \mathrm{y}\right.$ $\varepsilon_{\max }=12.57 \%$ ) están muy próximos a los límites exigidos

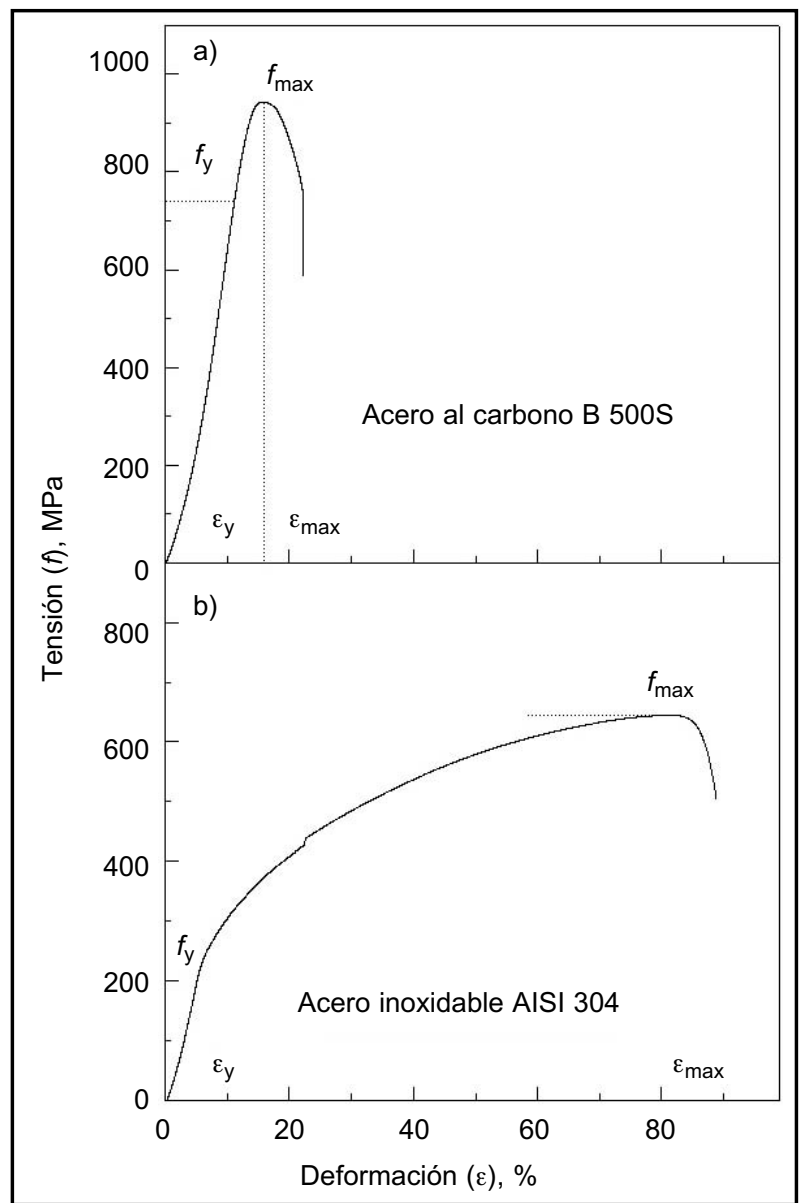

Figura. 2. Diagramas tensión deformación de barras de (a) acero al carbono B 500S, y (b) acero inoxidable AISI 304, sin escalón de cedencia.

Tabla 3

Composición química (\% peso ${ }^{a}$ ) de los tres tipos de acero ensayados, acero al carbono (tipo B500S), acero inoxidable AISI 304 y del nuevo acero inoxidable bajo en níquel.

\begin{tabular}{|c|c|c|c|c|c|c|c|c|c|c|}
\hline Material & $\mathbf{C}$ & $\mathbf{S i}$ & $\mathbf{M n}$ & $\mathbf{P}$ & $\mathbf{S}$ & $\mathbf{C r}$ & $\mathbf{N i}$ & $\mathbf{M o}$ & $\mathbf{C u}$ & $\mathbf{N}$ \\
\hline B 500S & 0.15 & 0.22 & 0.72 & $<0.01$ & 0.022 & 0.13 & 0.13 & - & 0.18 & - \\
\hline AISI 304 & 0.070 & 0.75 & 2.00 & 0.075 & 0.015 & 18.50 & 9.00 & - & - & - \\
\hline Bajo Níquel & 0.082 & 0.48 & 7.26 & 0.027 & 0.001 & 16.56 & 4.32 & 0.07 & 0.13 & 0.075 \\
\hline
\end{tabular}

a La diferencia es Fe. 
por la Instrucción EHE-08 (1,20 y 10\%, respectivamente), mientras que para el nuevo acero inoxidable bajo en níquel así como el AISI 304 los valores obtenidos son muy superiores a los exigidos por la EHE-08, siendo para el nuevo acero inoxidable bajo en níquel $\left(f_{\text {max }} / f_{\mathrm{y}}=\right.$ 2,22 y $\left.\varepsilon_{\max }=69,21 \%\right)$ y para el AISI $304\left(f_{\max } / f_{\mathrm{y}}=2,83\right.$ y $\left.\varepsilon_{\max }=78,57 \%\right)$.

La Figura 5 muestra los resultados de ductilidad obtenidos para el índice " $p$ " aplicando el criterio de acero equivalente según el criterio de Cosenza y col. (23). Puede observarse que los tres aceros ensayados ofrecen un valor muy superior para el índice " $p$ " de acero equivalente $(29,27$ para el acero inoxidable bajo en níquel, 48,29 para el AISI 304 y 1,74 para el acero al carbono B500S) que el valor exigido por la EHE-08, y los códigos CM-90 y EC-2 $(0,13 \leq p \leq 0,83)(26,28,29)$, en particular en una sección de EHA con AISI 304 se ha obtenido una capacidad de giro de 48,29/0,83 = 58,18 veces superior que el acero 500SD según la EHE-08 (26). Para el nuevo acero inoxidable bajo en níquel, la capacidad de rotación (evaluada

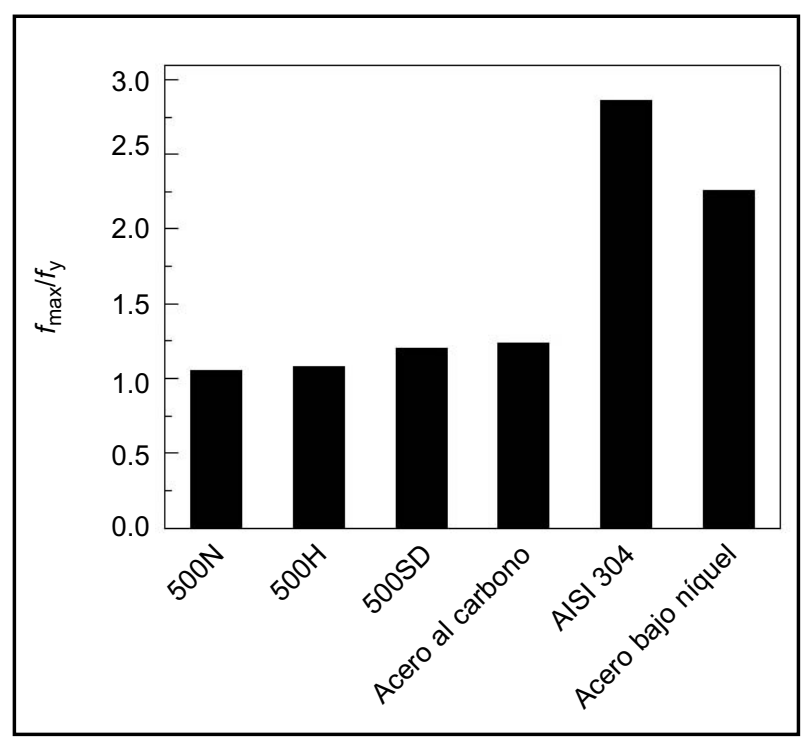

Figura. 3. Variación de la relación entre la tensión máxima $\left(f_{\max }\right)$ y la tensión en el límite elástico $\left(f_{\mathrm{y}}\right)$ para el acero al carbono, y los aceros inoxidables bajo en níquel y AISI 304.

Tabla 4

Resultados experimentales obtenidos mediante los ensayos de tracción. Acero al carbono B 500S, acero inoxidable AISI 304, acero inoxidable bajo en níquel; acero B 500SD, acero de alta ductilidad $500 \mathrm{H}$, acero de ductilidad normal $500 \mathrm{~N}$, tensión máxima ( $\left.f_{\text {max }}\right)$, tensión en el límite elástico $\left(f_{y}\right)$, módulo de Young $(E)$, deformación bajo carga máxima $\left(\varepsilon_{\max }\right)$; deformación en el límite elástico $\left(\varepsilon_{\mathrm{y}}\right)$, índice de Cosenza y col. $(\mathrm{p})$, índice de Creazza y col. $\left(\mathrm{A}^{*}\right)$, e índice de Ortega $\left(\mathrm{I}_{\mathrm{d}}\right)$.

\begin{tabular}{|c|c|c|c|c|c|c|c|c|c|}
\hline \multirow{2}{*}{ Material } & \multicolumn{9}{|c|}{ Parámetro } \\
\cline { 2 - 11 } & $\boldsymbol{f}_{\max } \mathbf{N ~ m m}^{-\mathbf{2}}$ & $\boldsymbol{f}_{\mathbf{y}} \mathbf{N ~ m m}^{-\mathbf{2}}$ & $\mathbf{E ~ N ~ m m}^{-2}$ & $\boldsymbol{\varepsilon}_{\max } \%$ & $\boldsymbol{\varepsilon}_{\mathbf{y}} \%$ & $\boldsymbol{f}_{\max } / \boldsymbol{f}_{\mathbf{y}}$ & $\mathbf{p}$ & $\mathbf{A}^{*} \mathbf{N ~ m m}^{-2}$ & $\mathbf{I}_{\mathbf{d}}$ \\
\hline B500S & 943 & 749 & 226065 & 12.57 & 0.33 & 1.26 & 1.74 & 15.83 & 84.82 \\
\hline AISI 304 & 639 & 226 & 133053 & 78.57 & 0.17 & 2.83 & 48.29 & 215.86 & 1767.31 \\
\hline Bajo níquel & 683 & 308 & 164996 & 69.21 & 0.19 & 2.22 & 29.27 & 172.55 & 1170.71 \\
\hline 500SD & 575 & 500 & 200000 & 10.00 & 0.25 & 1.20 & 0.83 & 4.87 & 86.80 \\
\hline 500H & 540 & 500 & 200000 & 5.00 & 0.25 & 1.08 & 0.34 & 1.27 & 39.52 \\
\hline 500N & 525 & 500 & 200000 & 2.50 & 0.25 & 1.05 & 0.13 & 0.37 & 18.45 \\
\hline
\end{tabular}

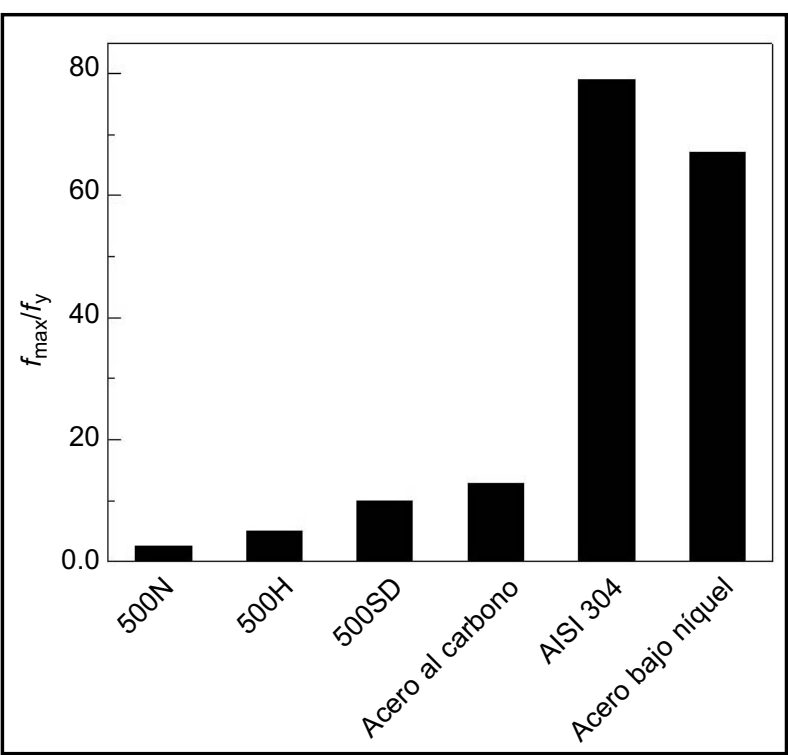

Figura. 4. Variación de la deformación bajo carga máxima $\left(\varepsilon_{\max }\right)$ para el acero al carbono, y los aceros inoxidables bajo en níquel y AISI 304.

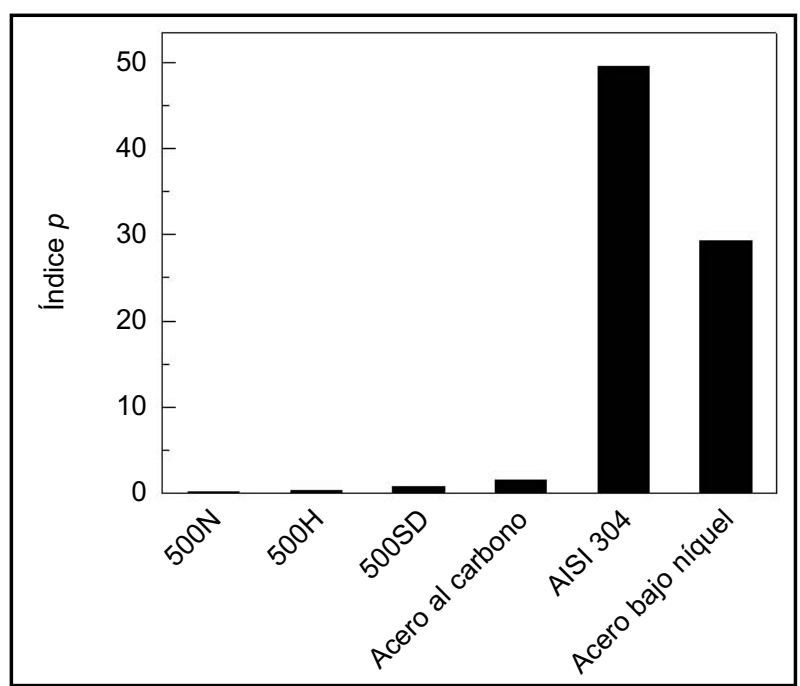

Figura. 5. Variación del índice " $p$ ", según el criterio de Cosenza y col. para el acero al carbono, y los aceros inoxidables bajo en níquel y AISI 304. 


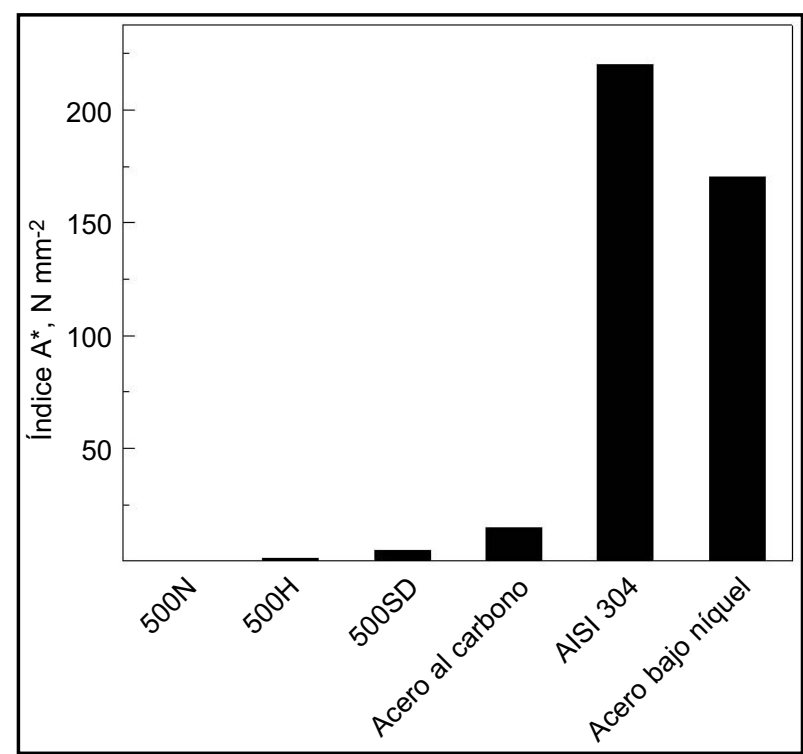

Figura 6. Variación del índice $A^{*}$, según el criterio de Creazza y col. para el acero al carbono, y los acero inoxidables bajo en níquel y AISI 304.

según la ecuación [1] ha sido de 29.27/0.83=35.26 veces superior al acero al carbono 500SD.

La Figura 6 presenta los valores de ductilidad resultado de aplicar el índice $A^{*}$ para acero equivalente de los tres aceros ensayados según el criterio de Creazza y col. (24). De forma análoga a los valores obtenidos aplicando el índice " $p$ " (Figura 5), tanto el nuevo acero inoxidable bajo en níquel como el AISI 304 presentan un valor del índice A $^{*}$ superior $\left(172,55\right.$ y $215,86 \mathrm{~N} \mathrm{~mm}^{-2}$, respectivamente) que el del acero al carbono $B$ 500S y 500SD reflejado en la EHE-08, y los códigos CM-90 y EC-2 $(26,28,29)$.

La Figura 7 muestra los resultados obtenidos aplicando el índice de tenacidad $\mathrm{I}_{d}$ para los tres aceros ensayados según el criterio de Ortega (25). El diagrama de la figura ha sido obtenido eliminando la influencia del límite elástico $\left(f_{y}\right)$. Se observa que el acero al carbono ensayado presenta un valor de $I_{d}$ inferior al valor del acero de referencia 500SD según la EHE-08 (26). El índice de tenaciadd $\mathrm{I}_{d}$ es mucho más estricto que los índices de ductilidad " $p$ " $y$ $A^{*}$ (Figuras 5 y 6 , respectivamente). Los aceros inoxidables ensayados cumplen las exigencias de la EHE-08, así como de los códigos CM-90 y EC-2. Se observa que el AISI 304 es ligeramente más dúctil que el nuevo acero inoxidable con bajo contenido en níquel.

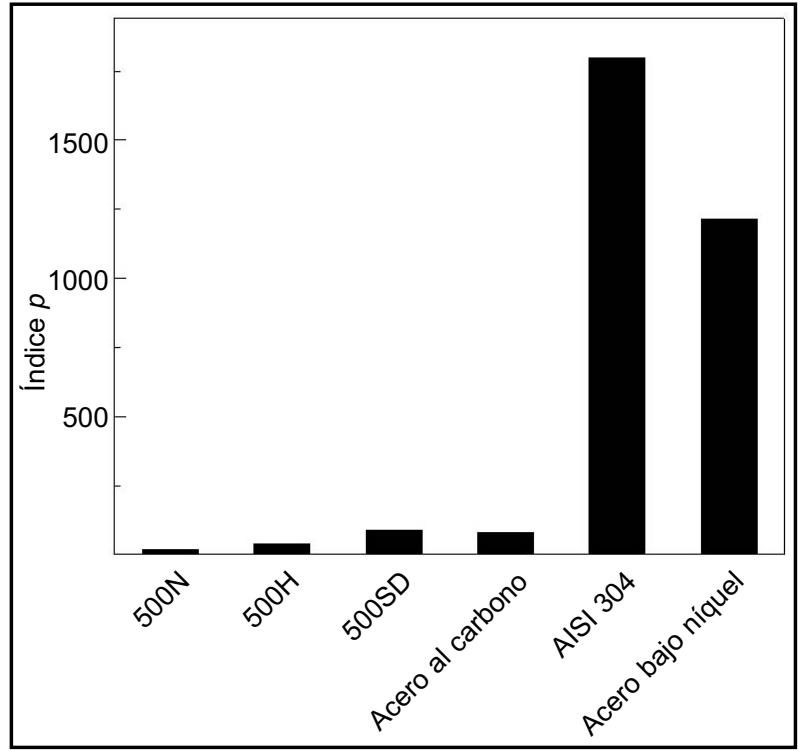

Figura 7. Variación del índice de tenacidad $\mathrm{I}_{d}$, según el criterio de Ortega para el acero al carbono, y los aceros inoxidables bajo en níquel y AISI 304.

\section{CONCLUSIONES}

El nuevo acero inoxidable satisface notablemente los criterios mínimos para los parámetros y $\varepsilon_{\max }$, exigidos por la Instrucción Española de Hormigón Estructural EHE-08, así como por los códigos europeos CM-90 y EC-2, siendo por tanto un buen candidato para ser utilizado como alternativa en armaduras para fabricar EHA.

El nuevo acero inoxidable bajo en níquel cumple las propiedades de ductilidad resultantes de aplicar los criterios de acero equivalente propuestos por Cosenza y col., Creazza y col. y Ortega. Tanto el nuevo acero inoxidable bajo en níquel como el AISI 304 presentan una mayor ductilidad que el acero al carbono convencional B 500S, aún siendo comparados con los aceros de los tipos 500SD, 500N y 500H.

\section{AGRADECIMIENTOS}

Los autores expresan su agradecimiento al proyecto BIA2008-05398 de la CICYT, por su apoyo económico y a la empresa ACERINOX, S.A., por proporcionar las muestras de acero inoxidable. D. M. Bastidas agradece al Programa Ramón y Cajal del Ministerio de Ciencia e Innovación la financiación de su contrato.

\section{BIBLIOGRAFÍA / BIBLIOGRAPHY}

(1) Page, C. L., Treadaway, K. W. J.: "Aspects of the electrochemistry of steel in concrete", Nature, no 297 (1982), pp. 109-115. http://dx.doi.org/10.1038/297109a0

(2) Flaga, K.: "Advances in materials applied in civil engineering", J. Mater. Process. Tech., no 106 (2000), pp. 173-183. 
(3) Committee Euro-International du Béton, Coating Protection for Reinforcement. CEB State of the Art Report, Thomas Telford Services Ltd, London (1995).

(4) Barris, C., Torres, L. I., Baena, M., Miás, C.: "Vigas de hormigón armado con barras de materiales compuestos". IV Congreso de la Asociación Científico-Técnica de Hormigón Estructural, Valencia, Spain (2008).

(5) Macias, A., Andrade, C.: "Corrosion rate of galvanized steel reinforcements in alkaline solutions. Part 2: SEM study and identification of corrosion products", Brit. Corros. J., no 22 (1987), pp. 119-129.

(6) Sánchez, M., Alonso, M. C., Cecílio, P., Montemor, M. F., Andrade, C.: "Electrochemical and analytical assessment of galvanized steel reinforcement pre-treatment with Ce and La salts under alkaline media", Cement Concrete Comp., n 28 (2006), pp. 256-266.

(7) Trabelsi, W., Cecílio, P., Ferreira, M. G. S., Montemor, M. F.: "Electrochemical assessment of the self-healing properties of Ce-doped silane solutions for the pre-treatment of galvanized steel substrates", Prog. Org. Coat., no 54 (2005), pp. 276-284. http://dx.doi.org/10.1016/j.porgcoat.2005.07.006

(8) García-Heras, M., Jiménez-Morales, A., Casal, B., Galván, J. C., Radzki, S., Villegas, M. A.: "Preparation and electrochemical study of cerium-silica sol-gel thin films", J. Alloys Comp., no 380 (2004), pp. 219-224.

(9) Mora, N., Cano, E., Polo, J. L., Puente, J. M., Bastidas, J. M.: "Corrosion protection properties of cerium layers formed on tinplate", Corros. Sci., no 46 (2004), pp. 563-578. http://dx.doi.org/10.1016/S0010-938X(03)00171-9

(10) Montoya, R., Aperador, W., Bastidas, D. M.: "Influence of conductivity on cathodic protection of reinforced alkali-activated slag mortar using the finite element method", Corros. Sci., no 51 (2009), pp. 2857-2862. http://dx.doi.org/10.1016/j.corsci.2009.08.020

(11) Miller, J. B.: European Patent Application, No. 90108563.9 (1990).

(12) González, J. A., Cobo, A., González, M. N., Otero, E.: "On the effectiveness of realkalization as a rehabilitation method for corroded reinforced concrete structures", Mater. Corros., no 51 (2000), pp. 97-103.

(13) Castellote, M., Llorente, I., Andrade, C., Turrillas, X., Alonso, C., Campo, J.: "In-situ monitoring the realkalization process by neutron diffraction: Electroosmotic flux and portlandite formation", Cement Concrete Res., no 36 (2006), pp. 791-800. http://dx.doi.org/10.1016/j.cemconres.2005.11.014

(14) Miranda, J. M., González, J. A., Cobo, A., Otero, E.: "Several questions about electrochemical rehabilitation methods for reinforced concrete structures", Corros. Sci., no 48 (2006), pp. 2172-2188. http://dx.doi.org/10.1016/j.corsci.2005.08.014

(15) Uy, B.: "Stability and ductility of high performance steel sections with concrete infill", J. Constr. Steel Res., no 64 (2008), pp. $748-754$.

(16) Pérez Quiroz, J. T., Terán, J., Herrera, M. J., Martínez, M., Genescá, J.: "Assessment of stainless steel reinforcement for concrete structures rehabilitation", J. Constr. Steel Res., no 64 (2008), pp. 1317-1324.

(17) Baddoo, N. R.: "Stainless steel in construction: A review of research, applications, challenges and opportunities", J. Constr. Steel Res., no 64 (2008), pp. 1199-1206.

(18) Castro, H., Rodríguez, C., Belzunce, F. J., Canteli. A. F.: "Mechanical properties and corrosion behaviour of stainless steel reinforcing bars", J. Mater. Process. Tech., no 143-144 (2003), pp. 134-137.

(19) Cramer, S. D., Covino, B. S., Bullard, S. J., Holcomb, G. R., Russell, J. H., Nelson, F. J., Laylor, H. M., Soltesz, S. M.: "Corrosion prevention and remediation strategies for reinforced concrete coastal bridges", Cement Concrete Comp., no 24 (2002), pp. 101-117.

(20) UNE 36-067-94 Standard, Alambres corrugados de acero inoxidable austenítico para armaduras de hormigón armado, Asociación Española de Normalización y Certificación (1994).

(21) ASTM A955/A955M-04 Standard, Deformed and plain stainless steel bars for concrete reinforcement, ASTM, USA (2004).

(22) BS 6744 Standard, Stainless steel bars for the reinforcement of and use in concrete. Requirements and test methods, BS, UK (2001).

(23) Cosenza, E., Greco, C., Manfredi, G.: "An equivalent steel index in the assessment of the ductility performances of the reinforcement", Bulletin d'Information, no 242, Ductility of Reinforced Concrete Structures, Committee Euro-International du Béton, Lausanne (1998).

(24) Creazza, G., Russo, S.: "A new proposal for defining the ductility of concrete reinforcement steels by means of a single parameter", Bulletin d'Information no 242 - Ductility of Reinforced Concrete Structures, Committee Euro-International du Béton, Lausanne (1998).

(25) Ortega, H.: Estudio experimental de la influencia del tipo de acero en la capacidad de redistribución en losas de hormigón armado, PhD Thesis, Polytechnic University of Madrid, Madrid, (1998).

(26) Comisión Permanente del Hormigón, Instrucción de Hormigón Estructural EHE-08, Ministerio de Fomento, Madrid (2008).

(27) Doñate, A.: Aceros con características especiales de ductilidad para hormigón armado, Calidad Siderúrgica, AENOR, Madrid (2000).

(28) CEB-FIP Model Code 90, Reinforcing steel, Commité Euro-International du Béton, Federation International of Prestressed, Bulletin d'Information No. 213/214, pp. 71-74, Thomas Telford, Services Ltd, London (1993).

(29) EN 1992-1-1:2004 Eurocode 2: Design of concrete structures, Part 1-1: General rules and rules for buildings, Lausanne (2004).

(30) Ductility Working Group from the Concrete International European Committee, Lausanne, (1998).

(31) Fabbrocino, G., Manfredi, G., Cosenza, E.: "Ductility of composite beams under negative bending: an equivalence index for reinforcing steel classification", J. Constr. Steel Res., no 57 (2001), pp. 185-202.

(32) Moreno, E., Cobo, A., Fernández Cánovas, M.: "Ductility of reinforcing steel with different degrees of corrosion and the equivalent steel criterion", Mater. Construcc., vol. 57, nº 286 (2007), pp. 5-18. 